\title{
DAMAGE DETECTION USING LEAST-SQUARES SINGULAR VALUE DECOMPOSITION METHOD AND ITS SENSITIVITY TO EXPERIMENTAL DATA
}

\author{
H. V. Duarte ${ }^{1}$, L. V. Donadon ${ }^{1}$, R. P. Ribeiro ${ }^{1}$ \\ ${ }^{1}$ Department of Mechanical Engineering, Engeneering School at the Federal University of \\ Minas Gerais, hvduarte@ufmg.br
}

\begin{abstract}
A singular value decomposition algorithm, SVD, is applied to frequency response function matrix to identify damage. A composite beam is used to generate vibration data for a healthy and damaged database. The method employed was named robust singular value decomposition as this procedure uses robust statistics to avoid outlier data, so the analysis can be done in a set of healthy and damaged data. Remarks are made on efficiency of the detection algorithm for different damage levels, and measurement techniques.
\end{abstract}

Keywords: Singular value decomposition, structural monitoring, laminated composite damage detection, piezoelectric transducer.

\section{INTRODUCTION}

The structural health monitoring has been improved due to the need to reduce conservative project and equipment maintenance costs, and due to an increasing use of new materials mainly in aerospace industry. The composite structures have a damage mechanism different from those of metals that they are replacing and failure detection need to be improved.

Changes in dynamic response of the structure are linked to a change in stiffness, mass or damping of the structure and this is the basic idea of structural health monitoring, [1]. Many techniques proposed to detect damage are based in changes in modal parameters, a comprehensive review on modal parameters-based damage identification can be found in Fan and Qiao [2].

The use of modal analysis on structural health monitoring is a natural consequence of its use on dynamic behavior of structure analysis. However, a major concern is that its validity is limited to linear structures [3].Vanlanduit [4] said that methods based on modal parameters are sensitive to changes in environment, operational conditions and structural uncertainties. In recent publications the proper orthogonal decomposition (POD) method was proposed aiming to remove the influence of these external factors [4] and Shane [1].

The proper orthogonal decomposition (POD) is a powerful multi-variate statistical method for data analysis aimed at obtaining low-order approximate descriptions of a highdimensional process, Wu et. al [5]. It is an essential technique for data reduction and feature 
extraction, and has been widely used in various areas including image processing, signal analysis, data compression, process identification, adaptive control, and many others [5].

The POD is an orthogonal transformation that decorrelates the signal components and maximizes variance. By this way the POD method reduce a large number of interdependent variables to a much smaller number of uncorrelated variables while retaining as much as possible of the variation in the original variables Kerschen et. al [3]. The discrete implementation of the proper orthogonal decomposition is the popular singular value decomposition,Kerschen [6] and $\mathrm{Wu}[5]$.

There are different implementations of the proper orthogonal decomposition method to structural health monitoring. Vanlanduit [4] proposed the robust singular value decomposition method (RSVD) that uses the frequency response functions (FRFs) of displacement, force, acceleration or other output as a basic data for the method.

Another method is the ultrasonic guided waves, in particular Lamb waves, that is aimed at plate-like structures, Konstantinidis et. al [7]. Its ability to detect defects have been demonstrated by published results. The measurements are made using high frequency PZT sensors. Being thin and light they can be bonded or embedded on the structure and are ideal to use in large laminated structures.

As the RSVD presented good results employing FRF (frequency response functions) this procedure was used to analyse the FRFs of an Aluminum 2024-T4 laminated plate. The measurements were made by using high frequency PZT sensors from healthy plate and from this plate with different delamination levels. The PZTs can be used to excite the structure or used as passive sensor to measure the vibration signal.

This work is closely related to implementation of the robust singular value decomposition method (RSVD) as published by Vanlanduit [4]. However the emphasis is shifted towards the experimental instrumentation and sensitivity verification of the method. The bonded PZT sensors allowed good repeatability of the measurements, essential to method, and the high frequency range obtained is not usually found in the bibliography. The main assumption is that smallest faults can be found by changes in highest mode frequencies. The objective is to use the instrumentation of one method to improve the ability to find failures inside the structure.

\section{DAMAGE DETECTION ALGORITHM}

The classical Least-Squares SVD-based damage detection can be outlined as follow. The database is obtained from frequency response function, FRF, a $M \times N$ matrix $H=\left[H_{1}, H_{2}, \ldots H_{N}\right]$ at $N$ specified conditions, each matrix $H_{n}$ has FRF data from each $P$ specified positions. $N=N_{u}+N_{d}$ where $N_{u}$ is the undamaged experimental FRFs from healthy structure and $N_{d}$ is from damaged ones. The damaged database $N_{d}$ must be greater than $N_{u}$. The sequence steps of the damage detection algorithm are presented below.

1- Computing the SVD of the $M \times N$ matrix $H=U S V^{H}$;

2- Putting all singular values $s_{k+1}, . . s_{N}$, from $S=\operatorname{diag}\left(s_{1}, s_{2}, . ., s_{N}\right)$, bellow the noise level equals zero, $S 1=\operatorname{diag}\left(s_{1}, s_{2}, . ., s_{k}, 0,0, . ., 0\right)$;

3- Using singular vectors $U$ and $V$ from original matrix synthesize matrix $H 1$ of rank $k$ $H 1=U S 1 V^{H}$; 
4- Computing residual matrix $E 1, E 1=H-H 1$;

5- Computing the variance $s$ of the residuals:

$$
s=\frac{1}{M N-1} \sum_{i=1}^{M} \sum_{j=1}^{N}\left(H_{i, j}-H 1_{i, j}\right)^{2}
$$

6- Computing the variance of each measurement $j$ (each column $j$ ):

$$
s_{j}=\frac{1}{M-1} \sum_{i=1}^{M}\left(H_{i, j}-H 1_{i, j}\right)^{2}
$$

7- Estimating the relative distance $d_{j}^{S V D}$ of each measurement $j$ to subspace computed by SVD:

$$
d_{j}^{S V D}=\frac{s_{j}}{s}
$$

As the relative distance $d_{j}^{S V D}$ is defined as a variance ratio it obeys a $\chi^{2}$ distribution. For a $\chi^{2}$ distribution with a confidence level of $(100-\alpha)=95 \%$ and for $\left(N_{n}-1\right)$ degrees of freedom or equal to 30 if $\left(N_{n}-1\right)>30$ [4], a threshold $T$ can be defined as

$$
T=\sqrt{\frac{\chi_{(100-\alpha)_{\left(N_{n}-1\right)}}^{2}}{2}}
$$

\subsection{The Robust SVD}

The problem with the LS-SVD based damage detection is that it is very sensitive to outliers in measurements Vanlanduit [4]. To avoid this the robust method was proposed, and it did not compute the whole database $H$, the SVD is computed only over $N / 2$ observations, the original matrix is re-synthesized and the distances and the error are computed. The solution is the small cost function from $N ! /(N ! / 2)^{2}$ possible combinations of the database. The steps of the RSVD routine are:

1- Construct a matrix $H_{R}$ from matrix $H$ with $L=N / 2$ combination columns from $N$ columns of the original matrix.

a- compute the SVD of the matrix $H_{R}=U_{R} S_{R} V_{R}^{H}$;

b- compute the extended right singular vector $V_{E}=U_{R}^{H} S_{R}^{-1} H$;

c- computing re-synthesized matrix $H_{S}$ using $H_{S}=U_{R} S_{E} V_{E}^{H}, S_{E}(i, i)=S_{R}(i, i) i=$ $1,2, \ldots, L$ and $S_{E}(i, i)=0$ if $i>L$;

d- Computing the residuals $E_{S}=H-H_{S}$;

e- Computing the variance $s$ of the residuals using the median absolute deviation $M A D$, as in [4], $s=M A D\left(E_{S}\right)$, where:

$$
\operatorname{MAD}\left(E_{S}\right)=1.4826 * \text { median }\left(\left|E_{S}-\operatorname{median}\left(E_{S}\right)\right|\right)
$$

f- Computing the variance of each measurement $j$ (each column $j$ ):

$$
s_{j}=\frac{1}{M-1} \sum_{i=1}^{L}\left(H_{i, j}-H_{S(i, j)}\right)^{2}
$$


g- Estimating the relative distance $d_{j}^{S V D}$ of each measurement $j$ to subspace computed by SVD:

$$
d_{j}^{S V D}=\frac{s_{j}}{s}
$$

h- compute the cost function $\kappa=\sum_{j}^{L}\left|d_{j}\right|^{2}$;

2- The smallest cost function $\kappa$ is taken as the RSVD solution, $H_{B}=H_{S}$. The variance $s$ and $d_{j}^{S V D}$ are computed following the procedures from $\mathbf{d}$ to $\mathbf{g}$ in the previous steps. As the relative distance $d_{j}^{S V D}$ is defined as a variance ratio it obeys a $\chi^{2}$ distribution. For a $\chi^{2}$ distribution. The threshold $T$ is the same as defined on Eq. 4 and there was employed the same confidence interval.

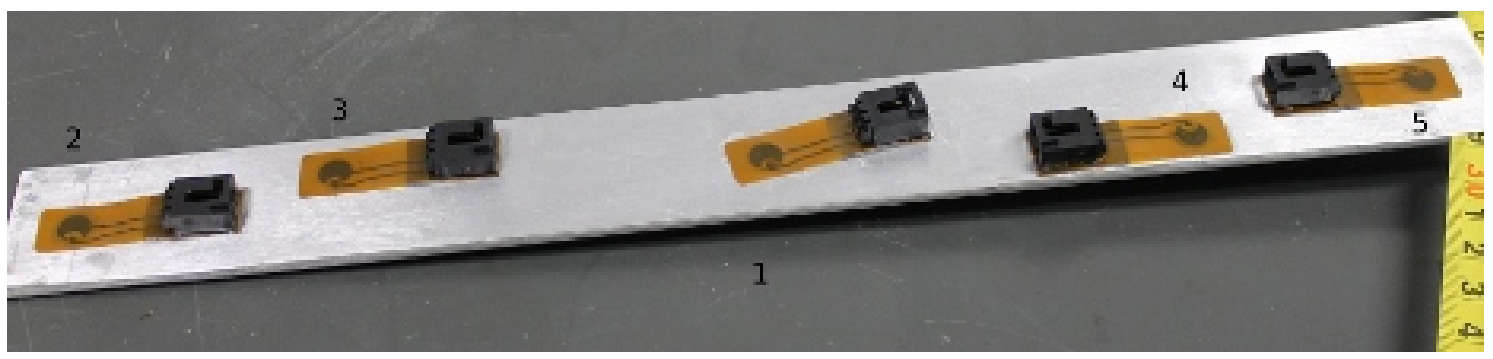

Figure 1. Laminate beam and PZT sensors.

\section{MATERIAL AND TESTING PROCEDURE}

The experimental procedure was done using dynamic response of a free-free laminate beam made by 4 Aluminum 2024-T4 $0.46 \mathrm{~mm}$ thick $(0.018 \mathrm{inch})$ plates and epoxy resin. The laminate beam has $300 \mathrm{~mm}$ length, $32.5 \mathrm{~mm}$ wide with a total thickness of $2.0 \mathrm{~mm}$. The measurement database was obtained from FRF response at 4 measurement points, 2, 3, 4 and 5, Fig. 1. Points 2 and 5 are on longitudinal axis and are symmetric about center, point 1, and point 3 and 4 are lateral and anti-symmetric. The measurement points 4 and 5 are the closest to failure area, Fig. 2, and both were used to excite the system as they provide high level vibration as active sensor than others. The point 1 was used as a reference point

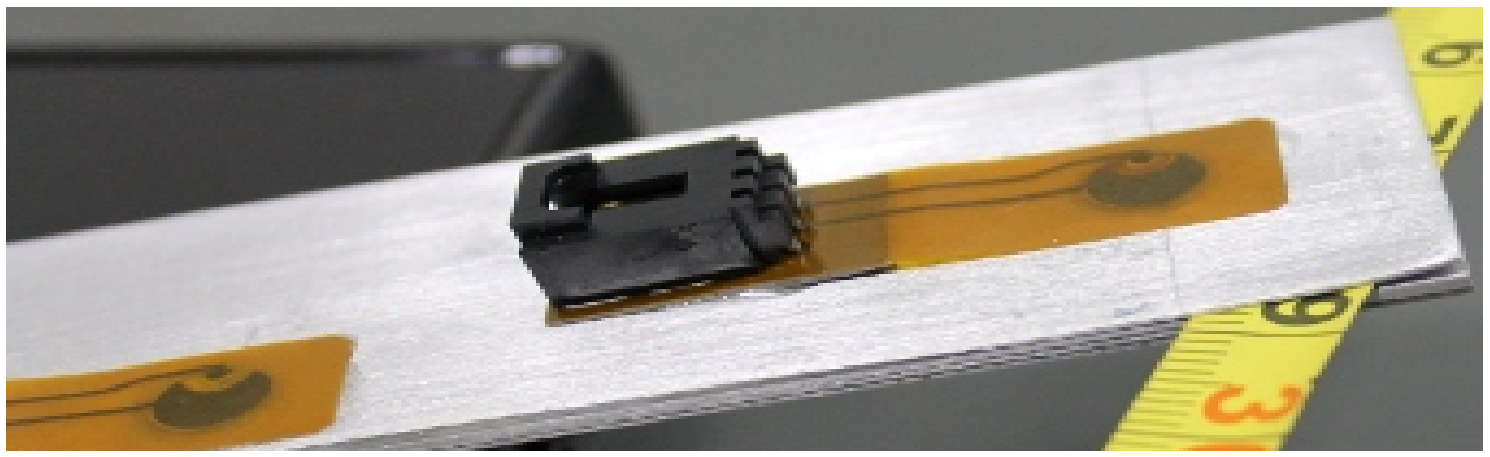

Figure 2. Laminate beam and damage detail with $2 \%$ of total area delaminated.

to obtain the FRFs as the measurement signal level were different from sensors to sensor. Using the passive signal of point 1 as reference for FRF do not change the results but reduce the dispersion level. A measurement run were made for the healthy laminate beam, another 
database were taken from this beam with a delaminate area of $195 \mathrm{~mm}^{2}, 2 \%$ of the total area, and another measurement run for the beam with a delaminated area of $504 \mathrm{~mm}^{2}, 5 \%$ of the total area.

The experimental data acquisition set was the PHOTON II Dynamic Signal Analyzer and all type of excitation signal employed was also generated by this equipment. The white noise, uniform random, sweep sine signals from the PHOTON II were amplified and sent to PZT 4 or 5, Fig. 1. All FRFs have units Volts/Volts (response/excitation) in a frequency range from 0 to $37500 \mathrm{~Hz}$. The PZTs sensors are from Acellent Technologies.

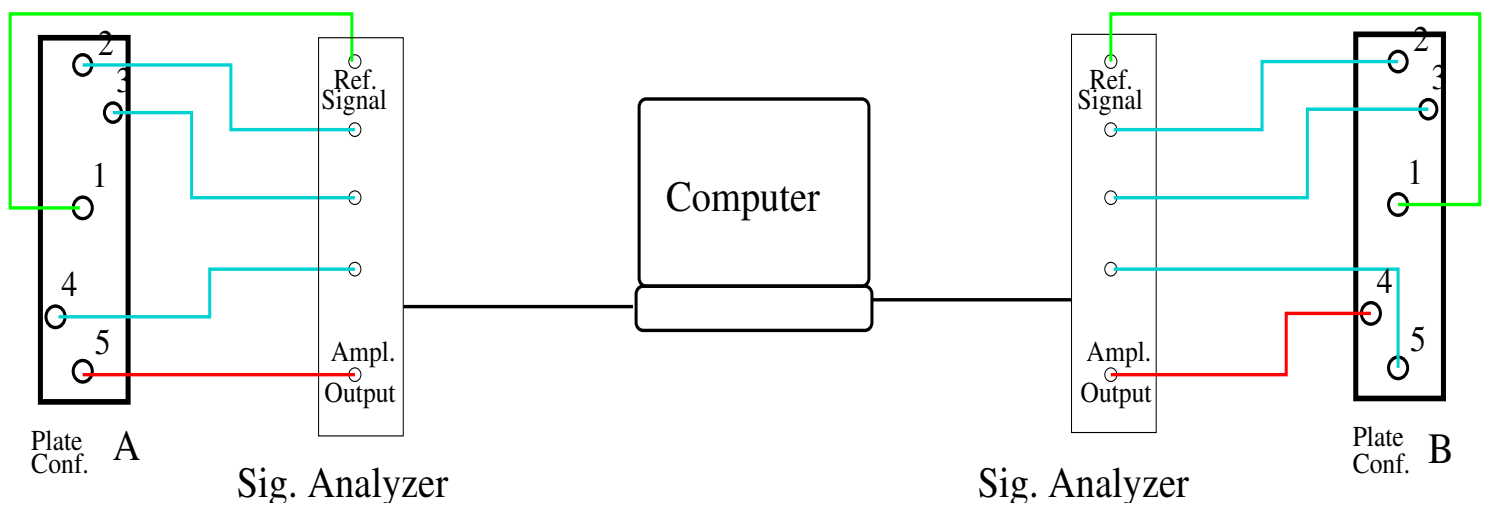

Figure 3. Measurement set. At left configuration $A$, and at right side configuration $B$.

\section{RESULTS AND DATA ANALYSIS}

Two experimental sets were made one of them the PZT 2, in longitudinal line, and lateral PZTs 3 and 4 are passive or measurement sensors, the PZT 5 was the active or excitation point receiving the signal from the amplifier, this configuration will be named configuration $A$, Fig. 3. The configuration $B$ was that where PZTs 2,3 and 5 were measurement sensors and the lateral PZT, at point 4 , was the the active sensor responsible for system excitation receiving the amplified signal, Fig. 3.

On Figure 4 there are from top, coherence for white noise excitation, below the coherence for uniform random followed by the sweep sine, at bottom the FRFs from which the coherences were obtained. As PZT sensors is for high frequency, the coherence does not exhibit good values for low frequency, as expected, and the FRFs had low amplitude too. The sweep sine excitation was set up to $0.91 \mathrm{sec} / \mathrm{sweep}$ and frequency range was from $1.0 \mathrm{kHz}$ to $37.5 \mathrm{kHz}$ and this set up behavior can be observed on graphic. The results are similar for coherence and for FRF for all kind of excitation signals and they were be considered good for frequencies above $5000 \mathrm{~Hz}$. The great majority of results presented in this work made use of sweep sine excitation as it presented a little better coherence results.

The results presented on Fig. 4 are from a 2\% damaged beam with excitation on lateral PZT close to fault, point 4, configuration $B$, and response measured on PZT 5, the point closest to damaged area. This point presented the worst behavior related to coherence as it had a low level response signal. The coherence signal was used here as criteria to discard some experimental results. In Figure 5 there are plots of FRFs, in logarithm scale, for measurement PZTs 2 and lateral PZTs 3 and 4, configuration $A$. The excitation was at point 5 . The results 

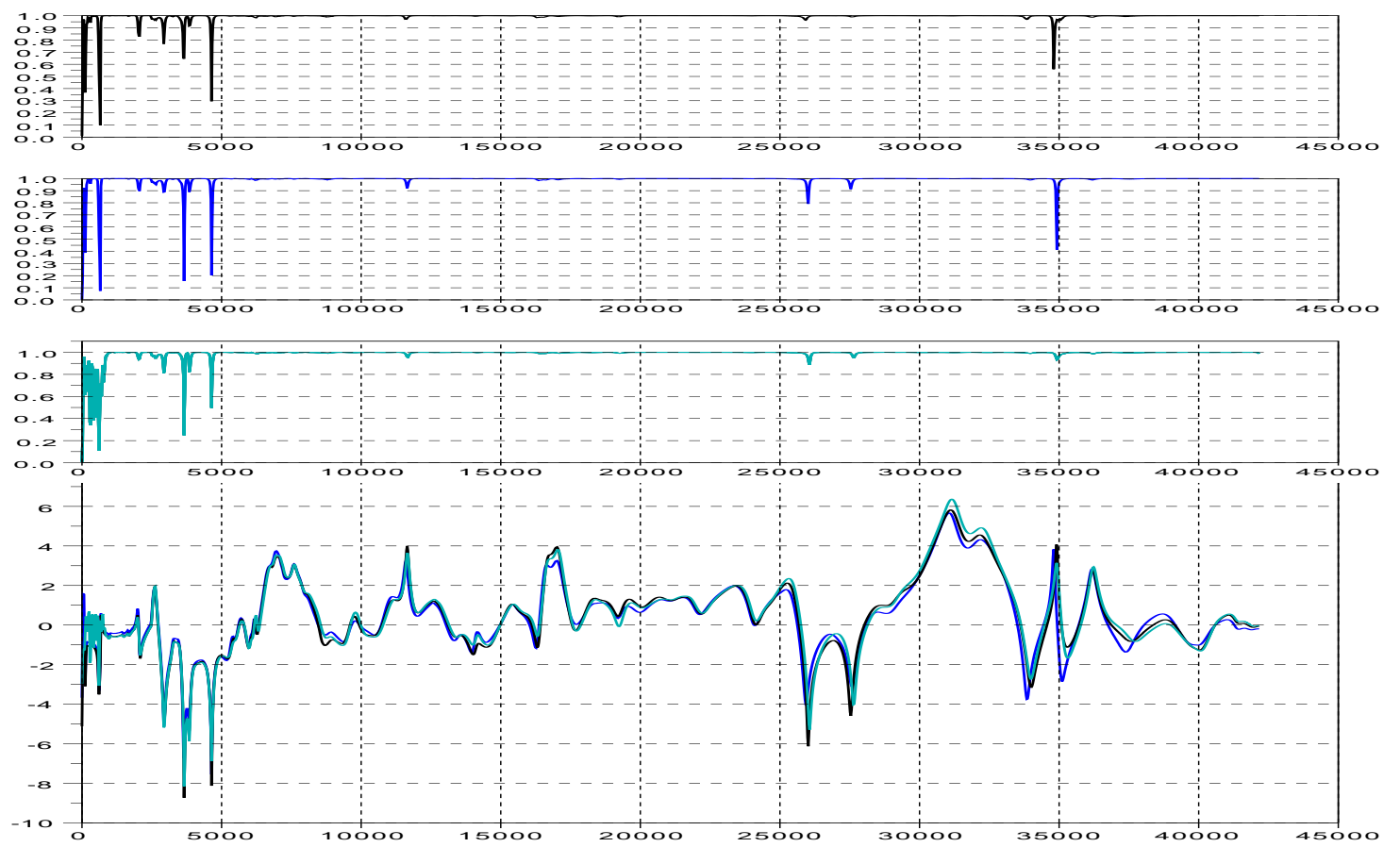

Figure 4. Coehence, from top to botton white noise, uniform random, sweep sine and the FRFs for $2 \%$ damaged plate.

are for the healthy structure. On Fig. 6 the results for points 2, 3, 5 with excitation point in the lateral PZT 4, configuration $B$.

Comparing $A$ and $B$ configurations the results showed differences in FRF for different excitation points. This behavior was expected as the excitation PZT were at different positions where different modes can be more easily excited. But for the same excitation position the measurement points 2, 3 and 4, configuration $A$, or 2, 3 and 5, configuration $B$ had similar FRFs with the same standing natural frequency mode peaks. The main different is the low signal level for points 3, 4 and 5 when compared to signal of point 2 .

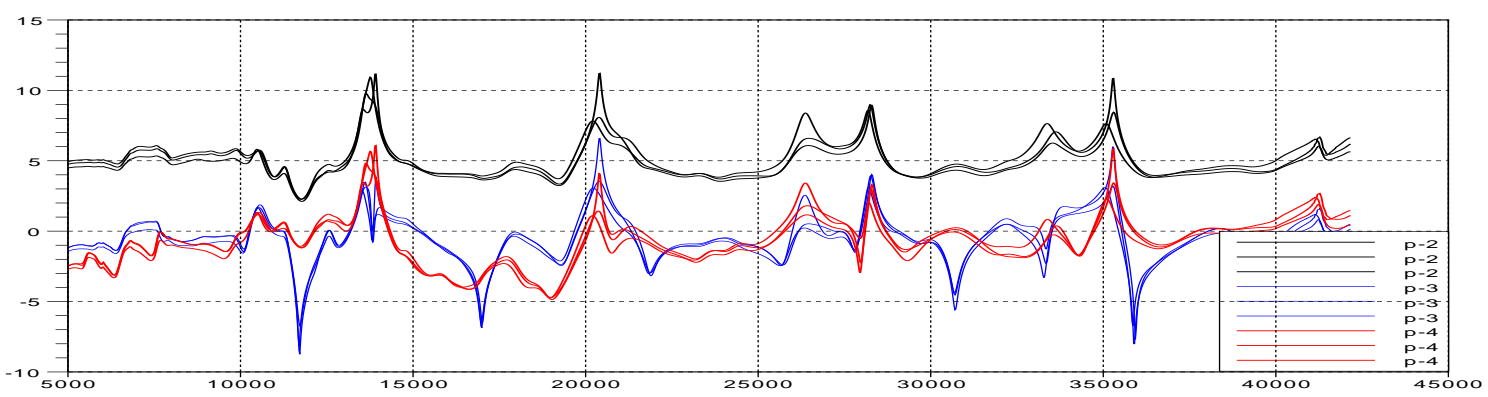

Figure 5. Measured FRFs for healthy structure in configuration $A$ (points 2,3 and 4). 


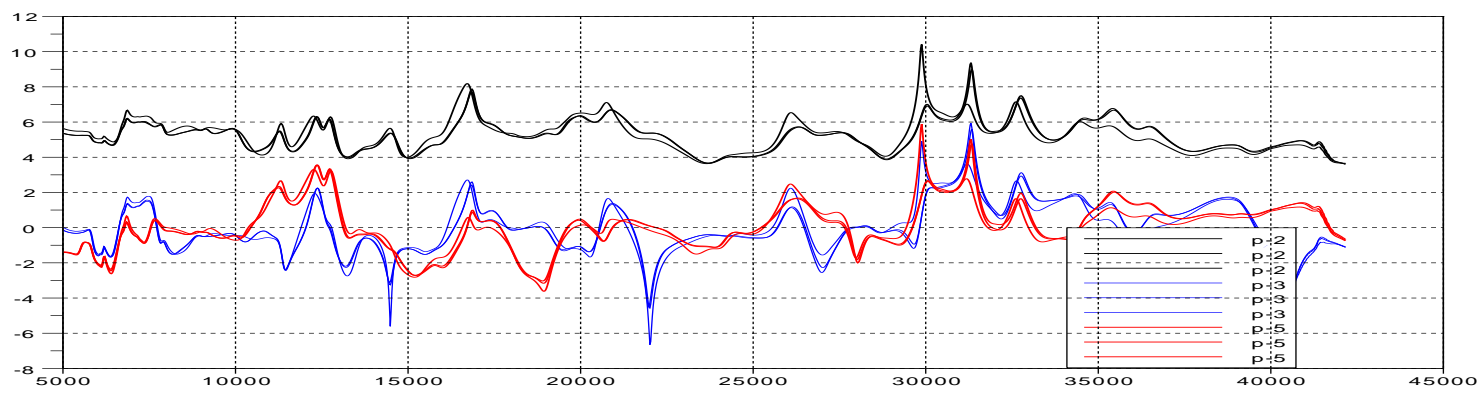

Figure 6. Measured FRFs for healthy structure in configuration $B$ (points 2,3 and 5).

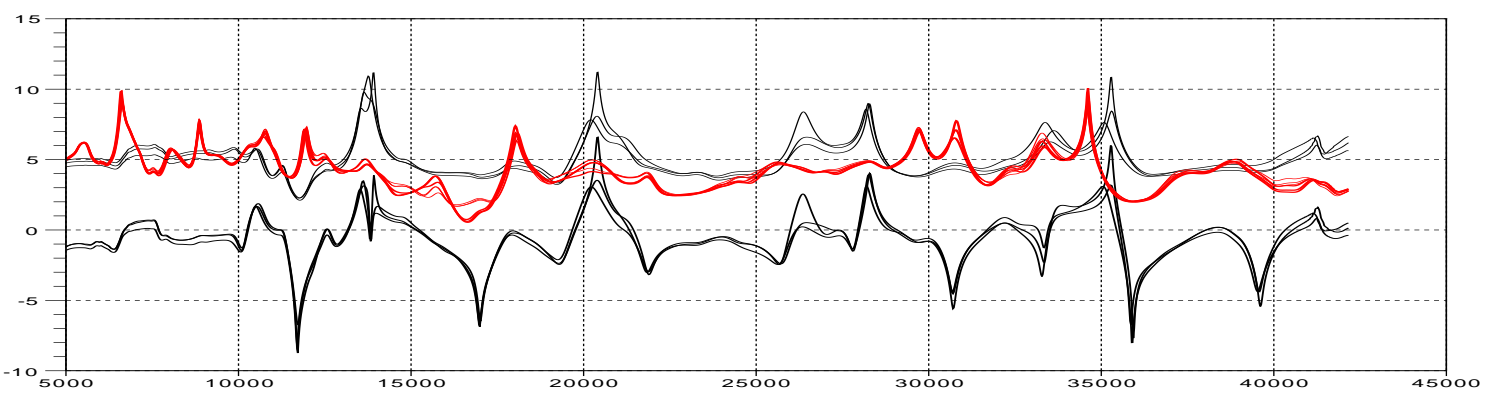

Figure 7. FRFs for points 2 and 3 configuration $A$. Healthy structure black lines, point 3 strong line, and red lines for $2 \%$ damaged one.

\subsection{Results for 2\% Damaged Structure in Configuration A}

The FRFs for the healthy and 2\% damaged structure are on Fig. 7 and Fig. 8 for excitation at PZT 5. On Fig. 7 there are only FRFs for measurement PZTs at positions 2 and 3, black lines for healthy and red lines for damaged structure. For the healthy structure, before being damaged, the FRFs for point 3 was plotted with a thicker line and has a low signal level than the FRF for point 2 as stated before. The response signal for points 2 and 3 were close and the FRF lines are almost not differentiable as the response signals level of point 3 raised. On Fig. 8 there are only FRFs for measurement points at position 4, black lines for healthy, and red lines for damaged structure.

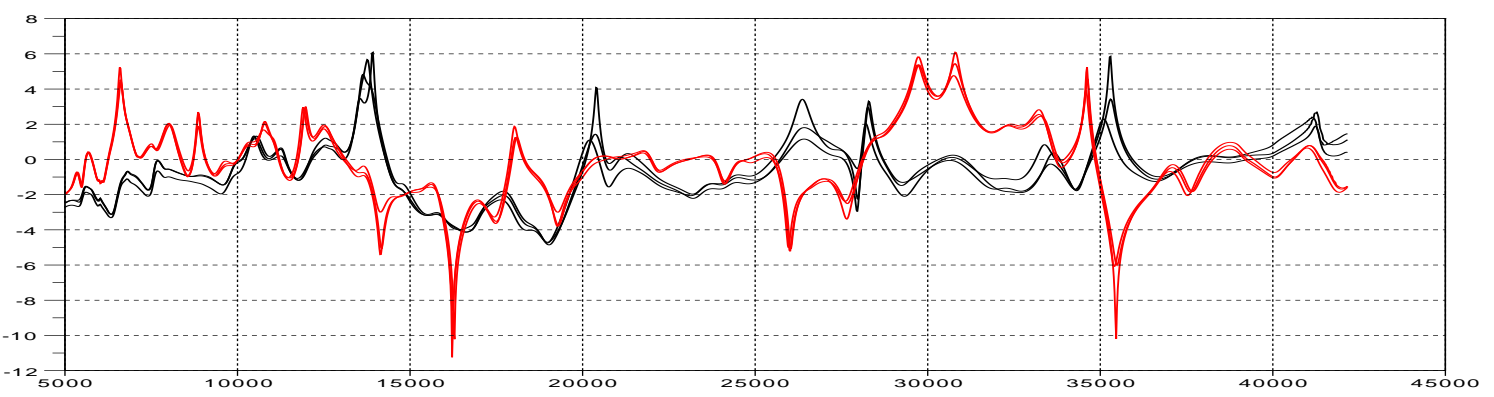

Figure 8 . FRFs for point 4 configuration $A$. Healthy structure black lines and red lines for $2 \%$ damaged one.

On Fig. 7 and Fig. 8 there are strong changes on the FRF shape for equivalent measurement points for all frequency range. There were no natural modes with the same or closed values for frequency or amplitude. As there are few measurement points it is impossible a 
modal analysis to identify each frequency mode and its change but there is no doubt that failure is responsible for changes.

Another remarkable change was the signal level on measurement PZT 3 that was attributed to stiffness change of the structure as there were no other experimental or measurement quality parameter that changed.

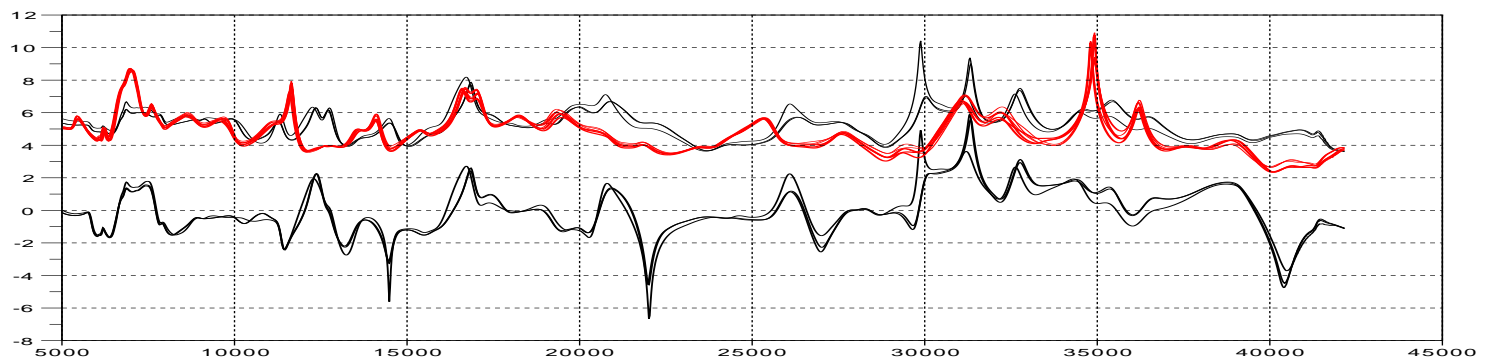

Figure 9. FRFs for points 2 and 3 configuration $B$. Healthy structure black lines, point 3 strong lines, and red lines for $2 \%$ damaged one.

\subsection{Results for 2\% Damaged Structure in Configuration B}

Comparing the FRF behavior between a healthy and 2\% damaged structure there are Fig. 9 and Fig. 10 for excitation at PZT 4. On Fig. 9 there are only FRFs for measurement points at positions 2 and 3, black lines for healthy, point 3 strong lines, and red lines for damaged structure as the response signal for points 2 and 3 had close response. On Fig. 10 there are only FRFs for measurement points at position 4, black lines for healthy and red lines for damaged structure.

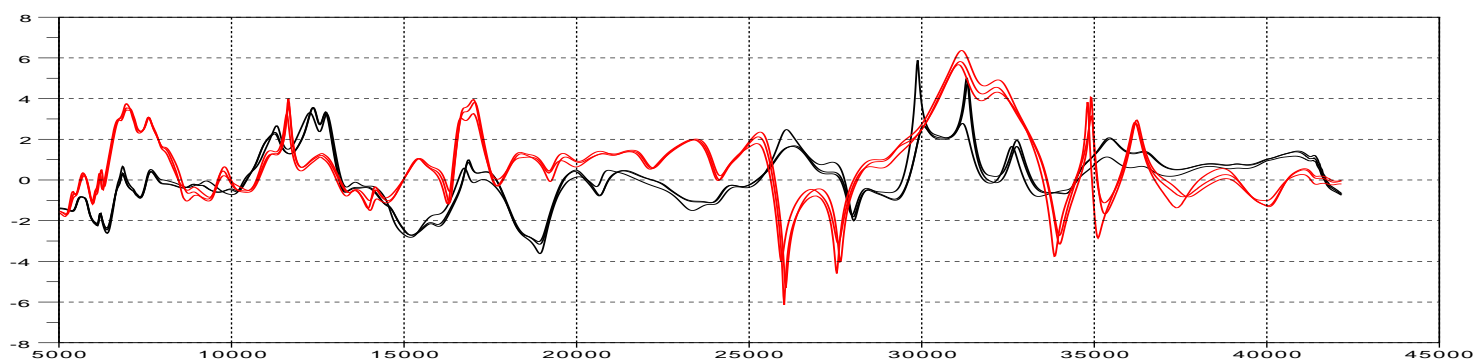

Figure 10. FRFs for point 5 configuration $B$. Healthy structure black lines and red lines for $2 \%$ damaged one.

A strong change in FRFs shapes can be observed in this configuration as observed in the previous one. But there are what could be seemed as similar measurement frequency behavior between healthy and damaged than one can observe for configuration $A$. As stated before, there are no confidence to make this analysis as there are no modal analysis to associate frequency and mode. The signal level of the passive PZT 3 had raised to a level close of the PZT2 as in previous case.

\subsection{Results for $5 \%$ Damaged Structure}

The FRFs for the healthy and 5\% damaged structure are on Fig. 11 and Fig. 12 for excitation at PZT 5. Following previous procedures, on Fig. 11 there are only FRFs for 


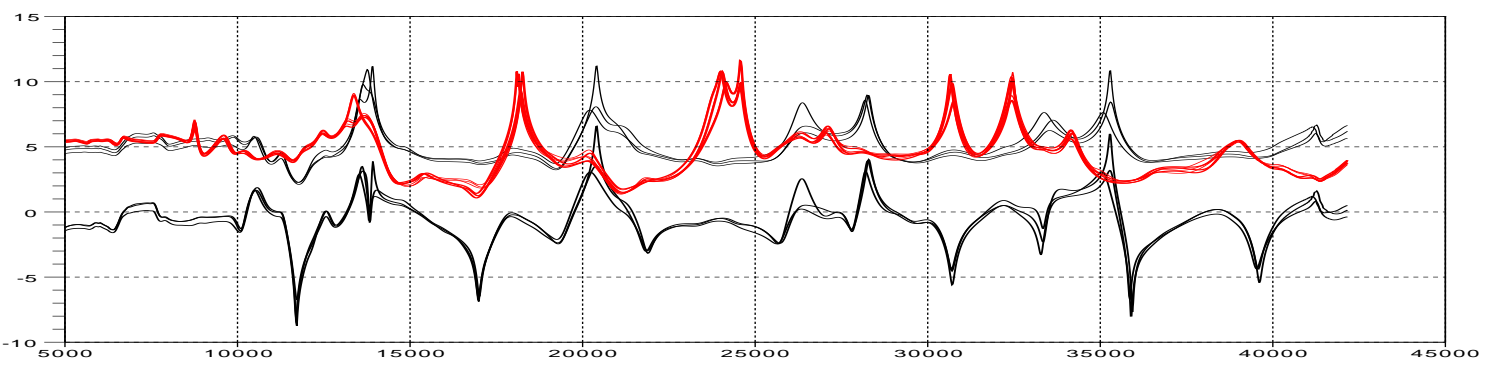

Figure 11. FRFs for points 2 and 3 configuration $A$. Red lines for 5\% damaged.

measurement points at positions 2 and 3, black lines for healthy and red lines for damaged structure. On Fig. 12 there are only FRFs for measurement at point 4. On Fig. 13 and Fig.

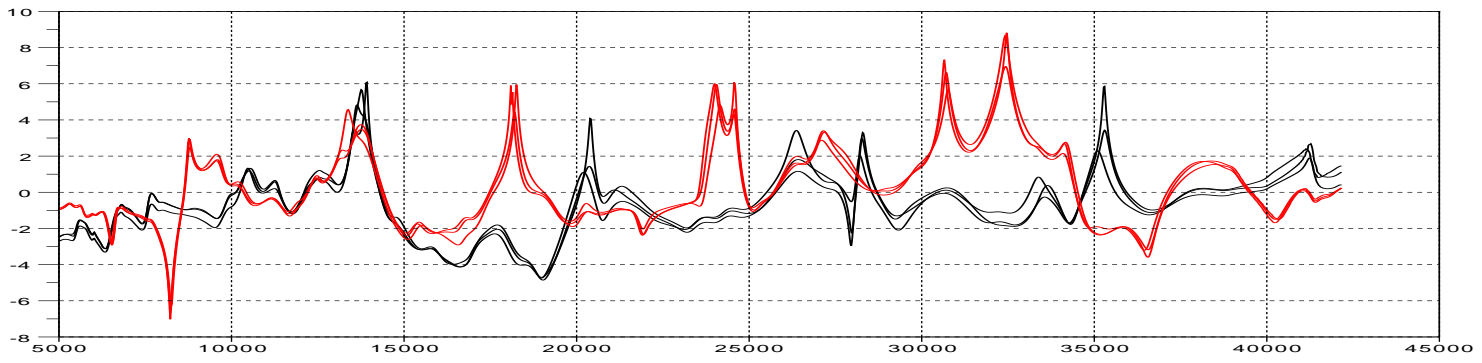

Figure 12. FRFs for point 4 configuration $A$. Red lines for $5 \%$ damaged.

14 are plotted the FRFs, PZTs at positions 2 and 3 for healthy and 5\% damaged structure in configuration $B$, for excitation at PZT 4 . On Fig. 14 FRFs for point 4 configuration $B$, black

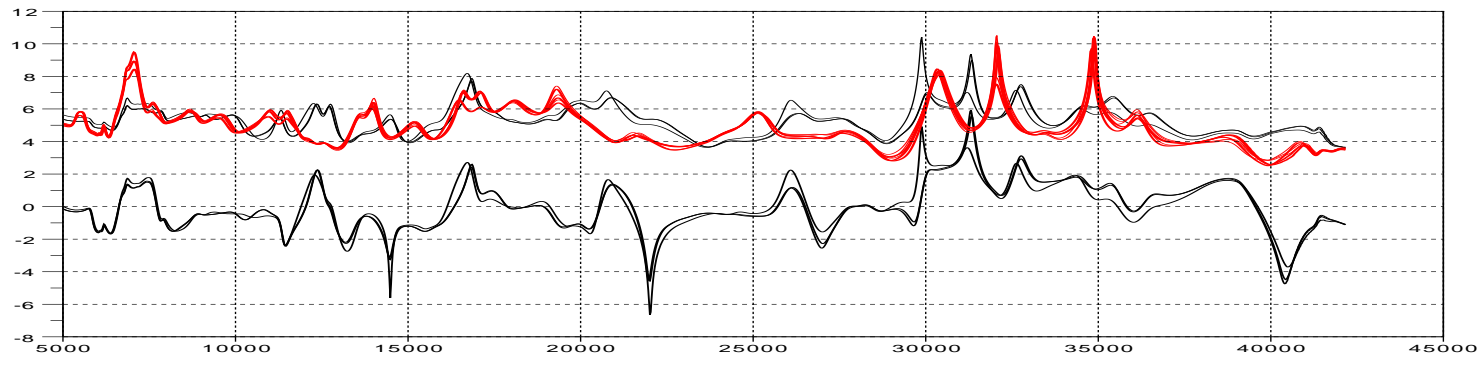

Figure 13. FRFs for points 2 and 3 configuration $B$. Red lines for $5 \%$ damaged.

lines for healthy and red lines for damaged structure. The same observations can be made about the FRFs for the 5\% damaged structure as stated for the $2 \%$ damaged. There were no natural modes with the same or closed values for frequency or amplitude and signal level on passive PZT 3 had the same level as the PZT 2 and the response curve of this sensors are close in both configurations and in both measurements.

On Fig. 15 are plotted the FRFs for 2 and 5\% damaged structures. This graphic was made only for configuration $B$ as the results were more close. The FRFs from measurement PZTs 2 and 3 for damaged structures are more similar than the healthy and 2\% delaminated composite beam. The main difference in FRFs are in two frequency modes inside the range from $29 \mathrm{kHz}$ to $33 \mathrm{kHz}$. The frequency modes are like and for $5 \%$ damaged structure, red lines, they show a highest amplitude at lowest frequency than the $2 \%$ damaged, black lines. Indeed 


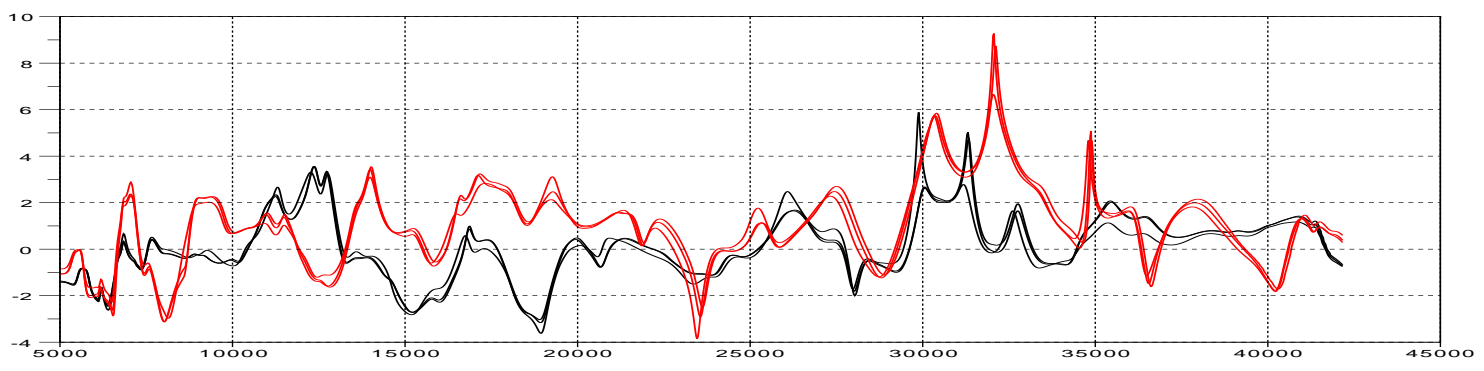

Figure 14. FRFs for point 4 configuration $B$. Red lines for $5 \%$ damaged.

the frequency modes for the $5 \%$ damaged presented low frequencies than the $2 \%$ damaged structure for the whole frequency band.

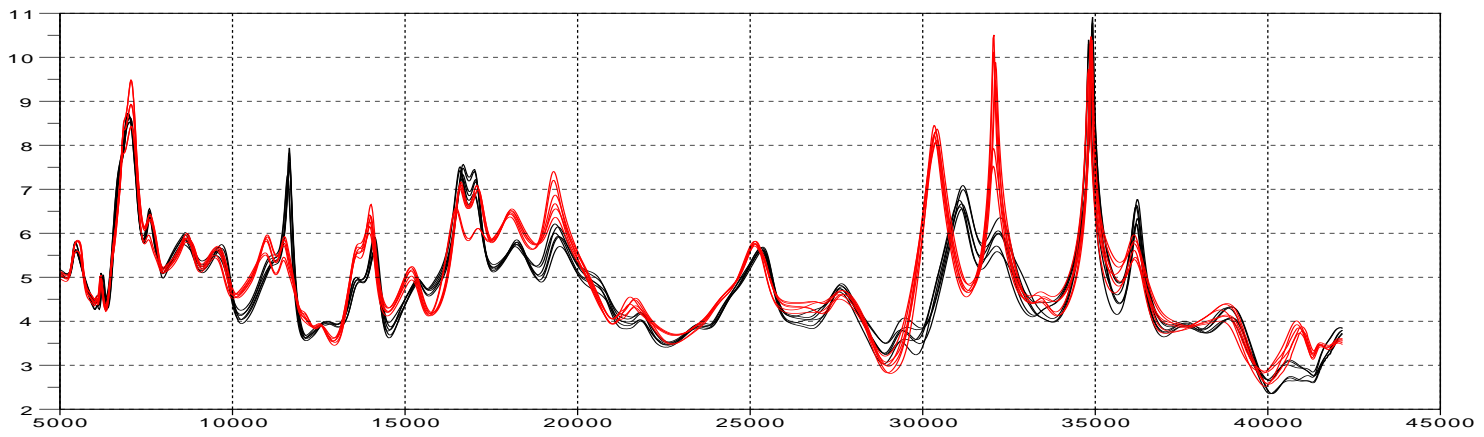

Figure 15. FRFs for PZT 2 and 3 configuration $B$. In black lines plots for $2 \%$ in red lines for $5 \%$ damaged structure.

The deviation of the 5\% and $2 \%$ delaminated structure from the healthy measurement were more expressive than expected. There is no an exact explanation for this behavior, but probably because the failure is close to a stressed lamina and although there is a small failure area it can lead to a expressive stiffness reduction. There is another explanation as the free lamina from the beam can hit the surface creating a nonlinear effect. Or both effects can be present.

\subsection{Numerical Analysis}

The numerical results were obtained following RSVD routine, the only change was related to the mechanism for minimizing the cost function, in this work was employed a genetic algorithm program. There was employed a Scilab algorithm, and the best result was for a population of 300 individuals, 400 couples, with crossover probability of $80 \%$, mutation probability of $30 \%$. Each element of the population was a reduced matrix $H_{r}$. The $H_{r}$ matrix was formed with 27 combinations of the 54 columns of the measurement matrix $H$. The matriz $H$ was composed by measurements from points 2,3 and 5 , configuration $B$. This measurements were for healthy, $2 \%$ and $5 \%$ damaged structure. The results are shown in Fig. 16.

It can be observed in Fig. 16 that the routine did not made a indication of damage. Comparing the data presented by Valanduit [4] and those presented here there are two major differences. The first is relative to proximity of the frequency peaks associated with the modes, 


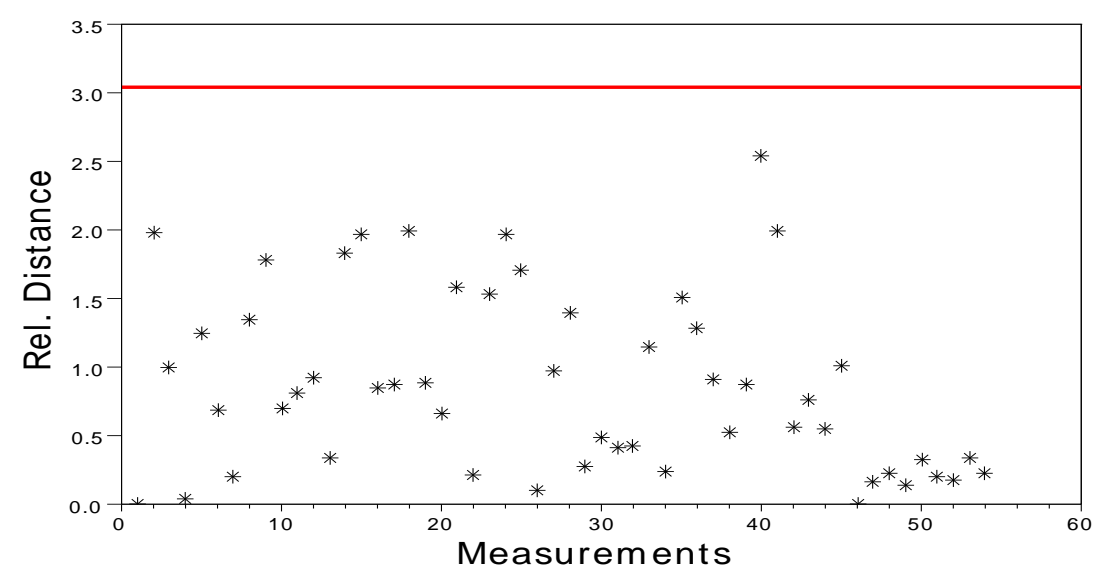

Figure 16. Threshold in red line and relative distances for each one of 54 experiments. Points 2,3 and 5 for healthy, $2 \%$ and $5 \%$ of delamination failure. Best cost function $\kappa$.

the Valanduit's results presented healthy and damaged peaks more closer than those observed here. The second aspect is related to different levels of the response signal, they are very different and it could be necessary a standardization of results.

\section{CLOSING REMARKS}

The results should be regarded as preliminary but show promising methods to be explored. The first one is the clear change in the FRFs for the healthy and damaged structure in high frequency. More measures are needed with other length to width ratio and changes the fault to inner layers.

The algorithm has been developed for identifying the first modes whose frequencies are generally little affected by small failures. Some corrections are needed as the normalization of FRFs. Another aspect is that the RSVD algorithm used 50\% of columns from $H$ to generate $H_{r}$, a matrix that will re-synthesize the original $H$ matrix as $H_{s}$, a matrix without the outliers for the best $\kappa$. But is not correct to suppose that the outliers always could be less than $50 \%$ of the measurement data as a datum from a damaged structure might be considered as a outlier. 


\section{REFERENCES}

[1] Shane C., Jha R., "Proper orthogonal decomposition based algorithm for detecting damage location and severity in composite beams". Mechanical Systems and Signal Processing, 25,1062-1072, 2011.

[2] Fan W., Qiao P., "Vibration-based Damage Identification Methods: A Review and Comparative Study". Structural Health Monitoring, 10(1),83-111, 2011.

[3] Kerschen G., Golival J.C., Vakakis A.F.,Bergman L.A., "The method of proper orthogonal decomposition for dynamical characterization and order reduction of mechanical systems: an overview". Nonlinear Dynamics, 41,147-169, 2005.

[4] Vanlanduit S., Parloo E., Cauberghe B., Guillaume P., Verboven P., " A robust singular value decomposition for damage detection under changing operating condtions and structural uncertainties". Journal of Sound and Vibration, 284,1033-1050, 2005.

[5] Wu C., Liang Y., Lin W., Lee H., S.P. L., "Letter to the editor: A note on equivalence of proper orthogonal decomposition methods". Journal of Sound and Vibration, 265,11031110, 2003.

[6] Kerschen G., Golinval J.C., "Physical interpretation of the proper orthogonal modes using the singular value decomposition". Journal of Sound and Vibration, 249(5),849-865, 2002.

[7] Konstantinidis G., Drinkwater B. W., Wilcox P. D., "The temperature stability of guided wave structural health monotoring system". Smart Materials and Structures, 15,967-976, 2006. 